\title{
DEVELOPMENT OF A SURGICAL NAVIGATION ROBOT USING IMAGE SERVO TRACKING TECHNIQUES
}

\author{
Chung-Hsien Kuo, Yu-Lin Tsai, Fang-Ghun Huang, Ming-Yih LeE
}

Department of Mechanical Engineering, Chang Gung University, Tao-Yuan, Taiwan

\begin{abstract}
This paper presents the development of surgical navigation robot, which is designed based on the image servo tracking technique. The surgical navigation robot plays an important role to the computer aided surgery (CAS), since it provides the patient's space coordinate during surgical navigation stage in real-time. Especially, the proposed active image servo tracking based surgical navigation robot can track the surgical marker that is mounted on the patient's hard tissue of the surgical position, so that the navigation space can be automatically extended. The proposed surgical navigation robot system consists of the in-lab designed dual-axis eye-in-hand robot (EIHR), digital signal processor (DSP) based EIHR motion controller, image servo tracking and positioning software. The EIHR robot is composed of the dual-axis robot mechanics and two CCD cameras, which acts as the stereoscopic image capture platform. The DSP based EIHR motion controller is designed to control the EIHR robot in a desired velocity, so that the surgical marker can be dynamically tracked. The image servo tracking and positioning software is the kernel of this work, and it is composed of image capture and recognition module, fuzzy logic based surgical marker tracking module and surgical marker positioning module. The image capture and recognition module is developed to abstract the image features of the surgical marker. The intelligent fuzzy logic based surgical marker tracking module is capable of generating robot's control command so that the surgical marker can be tracked within the desired target window. In addition, the surgical marker positioning module is used to calculate the surgical marker's space coordinates in terms of the features of stereoscopic images and the robot's posture. Finally, the prototype had been finished, and the experiment results showed that proposed EIHR achieved $1.7 \mathrm{~mm}$ position error within $500 \mathrm{~mm}$ operation range.
\end{abstract}

Biomed Eng Appl Basis Comm, 2004 (June); 16: 120-132.

Keywords: surgical assisted robot, eye-in-hand robot, stereoscopic surgical marker positioning, image servo tracking.

\section{INTRODUCTION}

In recent years, the $3 \mathrm{D}$ navigation systems had been successfully applied to the computer aided surgery (CAS) [1, 3-4, 9]. For example, Dohi et al. [3]

Received: Feb 16, 2004; Accepted: Mar 25, 2004

Correspondence: Ming-Yih Lee, Professor

Department of Mechanical Engineering, Chang Gung

University, Tao-Yuan, Taiwan

E-mail: leemiy@mail.cgu.edu.tw proposed a computer aided surgery system in 1990 . The proposed CAS was developed based on the surgical simulation and planning. The threedimensional graphic reconstruction was also implemented. A clinical trail of this system for a surgical planning and a prognosis of the liver carcinoma were discussed. The results showed a great possible use in the abdominal surgery.

Birkfellner et al. [1] developed a modular software system for the computer-aided surgery. This paper reported its first application in the oral implantology. The authors presented a programming 
environment using the high-level image processing library module that can connect with the position measurement device to perform the patient-to-image registration procedures. This environment could be applied to the navigation system for computer-aided implant dentistry. Based on the experimental trials, the overall target registration error (TRE) was found to be $1.2 \pm 0.3 \mathrm{~mm}$ in first phantom studies. In addition, the experiment took less than $5 \mathrm{~min}$ of navigation to find the target.

Edwards et al. [4] proposed the design and evaluation of the microscope-assisted guided interventions (MAGI) systems. The surgical navigation tasks consisted of the calibration of the optical system, registration of preoperative images to the patient, tracking of patient, and display using a suitable visualization scheme. In addition, the microscope calibration process can be automatically executed. The bone-implanted markers for registration and the incorporated locking acrylic dental stent (LADS) for patient tracking were introduced. Consequently, the LADS provided a less-invasive registration device with mean target error of $0.7 \mathrm{~mm}$ in volunteer experiments. Phantom accuracy was 0.3 to $0.5 \mathrm{~mm}$ and clinical overlay errors were 0.5 to $1.0 \mathrm{~mm}$ on the bone fiducials and 0.5 to $4 \mathrm{~mm}$ on target structures.

Kuo et al. [9] proposed a computer aided retained auricular prosthesis surgery system in 2003. In this work, a $3 \mathrm{D}$ digitizer was used to measure the coordinate and posture of the patient. The patient's coordinates were further transformed to the coordinates of the computer model. In addition, a sound-based navigation system was developed to guide the surgeon to reach the implant positions that were identified at the pre-surgery stage. Finally, the clinical validation of the retained auricular prosthesis surgery of the microtia children was introduced.

Based on the previous works, the surgical navigation system plays an important role to the CAS systems. Hence, this paper presents a stereoscopic vision based surgical navigation system using the EIHR robot. In order to capture the images of the freely moving object, a two-axis image servo controlled robot arm was designed. Two charge coupled device (CCD) cameras were mounted on the end effector of the robot arm as the "eye-in-hand" image tracking architecture. The images of the specially designed "three-ball" simulated surgical marker can be captured using the image grab card. By recognizing the radiuses and ball centers of the surgical marker, the space coordinate and orientation of the surgical marker can be calculated.

On the other hand, this work also developed an inlab designed digital signal processor (DSP) based servo motion controller and its peripheral control circuits to control the motion of the EIHR robot.
Meanwhile, the host computer and the DSP motion controller can communicate with each other using the serial communication interface. The fuzzy logic was developed in this work to track the surgical marker to automatically extend the navigation space in realtime. And, the DSP based motion controller can accept the commands from the image servo controller so that the freely moving object can be tracked dynamically.

The overall system was developed using the techniques within the medical mechatronics. The servo motion controller used the Texas Instruments (TI) TMS 320 C31 DSP processor [2, 13-14]; the image acquisition used the National Instruments (NI) IMAQ 1409 image grab card [16]; and the software programs of image recognition, space coordination calculation, fuzzy logic implementation, robot host controller were developed using the Microsoft Visual $\mathrm{C}++[8]$. The hardware and software components were properly integrated to achieve the desired purposes. The prototype of the image servo tracking based surgical navigation robot was initially validated using a mechanical 3D digitizer arm [9]. The results showed that the proposed system tracked the surgical marker in the target window so that the space coordinates can be calculated, and the positioning error is about $1.7 \mathrm{~mm}$ within $500 \mathrm{~mm}$ operation range. Finally, the techniques used in this system are illustrated in the following sections, and they are summarized as:

1. EIHR robot development.

2. DSP based servo motion controller development.

3. Stereoscopic image based surgical marker recognition and positioning module development.

4. Fuzzy logic based image servo tracking module development.

5. Space coordinates calculation module development.

6. System integration and window-based integrated program development.

7. System validation using the $3 \mathrm{D}$ mechanical digitizer arm.

\section{METHODS}

\subsection{System Development Architecture}

The proposed surgical navigation robot consists of the in-lab designed dual-axis eye-in-hand robot arm, robot motion controller, image capture and recognition module, fuzzy logic based simulated surgical marker tracking module, simulated surgical marker positioning module, and PC based host computer. The developed components are categorized into the hardware and software components as shown in Fig. 1. 


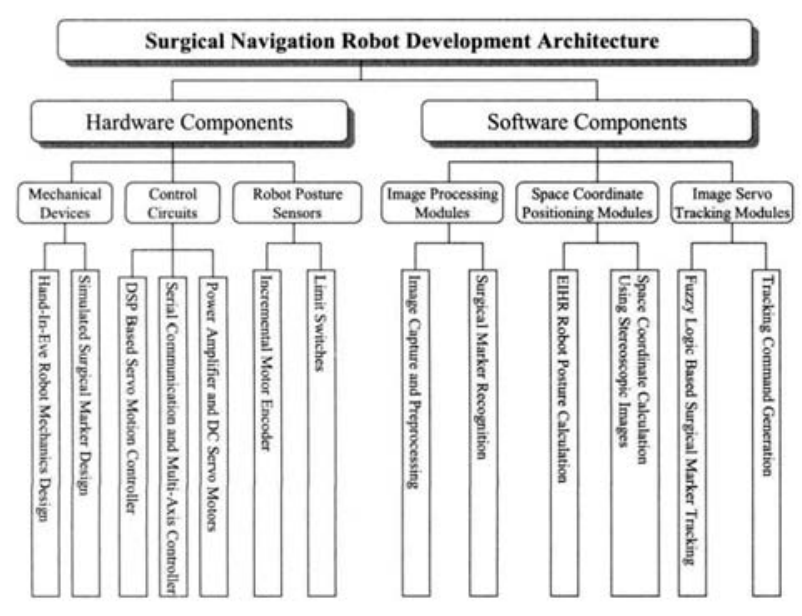

Fig. 1 Surgical navigation robot prototype development architecture

The hardware components consist of the mechanical devices, control circuit and robot posture feedback sensors. For the mechanical devices, the simulated surgical marker is designed to identify the patient's orientation. In addition, a two-axis hand-ineye robot mechanics is designed to setup the stereoscopic CCD cameras so that the freely moving surgical marker can be automatically tracked. For the control circuits, the DSP based PID controller is designed to control the angular velocities of the EIHR robot. Simultaneously, the angular positions can also be controlled. The serial communication and multi-axis controller is responsible for coupling with the DSP based motion controller to distribute the control signals to each axis and to establish the communication channel between the DSP motion controller and the host computer. The linear power amplifier is used to drive the DC servomotors. For the robot posture sensors, the incremental motor encoders were used to count the motor's angle and calculate angular velocity so that they can feedback to the DSP motion controller. In order to define the robot's home position and to limit the robot in the predefined workspace, the limit switches were used for each axis.

The software components consist of the image processing, space coordinate positioning, and image servo tracking modules. For the image processing modules, the stereoscopic images are acquired using the image grab card. The acquired images are further processed, and then the surgical marker can be recognized in terms of the centers and radiuses of the three-ball structure images. For the space coordinate positioning module, the recognized centers of the stereoscopic images can be used to calculate 3D space coordinate of the balls mounted on the surgical marker. Since the obtained $3 \mathrm{D}$ space coordinate is relative to the CCD camera platform, the space coordinate relative to the EIHR base must be computed from the robot's posture. The robot's posture can be calculated in terms of the forward kinematics [11]. In order to extend the navigation space automatically, the image servo tracking module was developed using the fuzzy logic to maintain the images of the surgical markers in the target window of the stereoscopic camera. Finally, based on the system development architecture, the operation flow of the system components can be also described in Fig. 2.

\subsection{Development of EIHR Robot}

In this paper, the eye-in-hand-robot was used to setup the stereoscopic CCD cameras. The EIHR robot is a two-axis motor-driven active robot arm. The EIHR was designed using the Pro-E software initially, and computer aided design (CAD) picture is shown in Fig. 3 . In addition to the stereoscopic CCD cameras, two DC servomotors with timing belt and gear trains were designed to drive the EIHR smoothly. The limit switches were designed to restrict the navigation robot in a predefined workspace. In order to acquire the angular positions and velocities of these two DC servomotors, the incremental encoders are coupled at the end of the DC servomotors. The assembled axes with timing belt, gear train, limit switches and encoders are shown in Fig. 4. Table 1 shows the mechanical and electrical specifications of the EIHR. Fig. 5 shows the photo of finally assembled EIHR robot with CCD cameras.

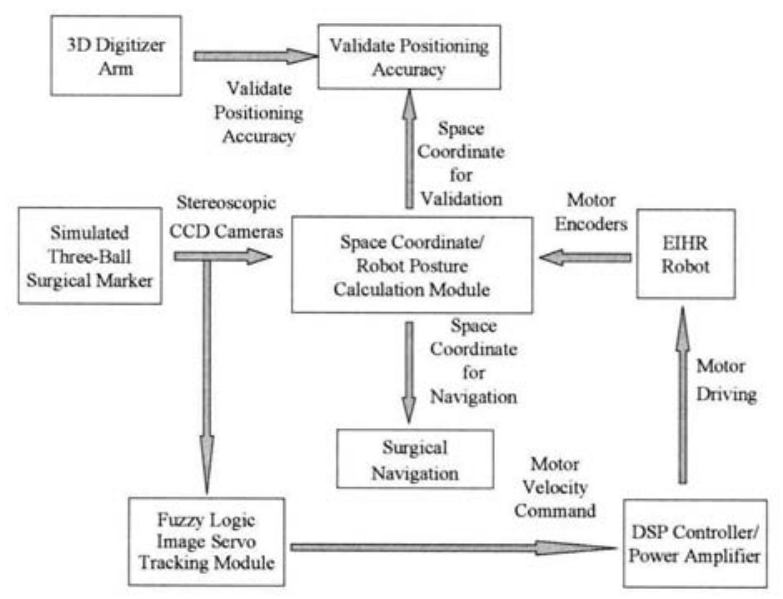

Fig. 2 Operation flow of system components 


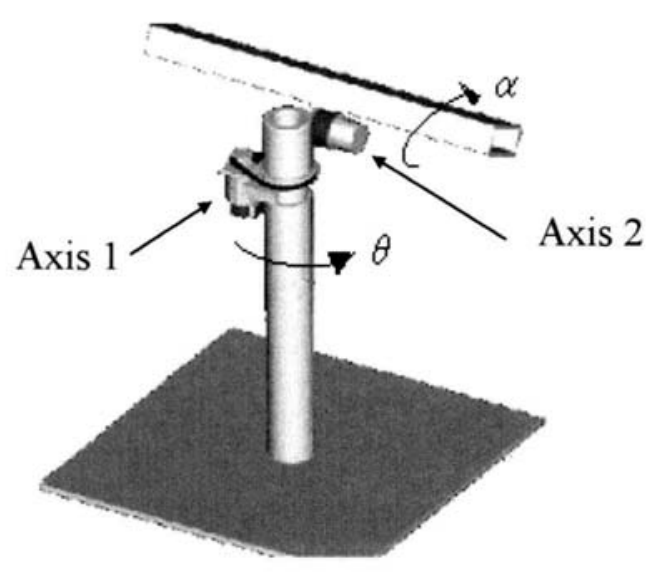

Fig. 3 Design of EIHR robot using Pro-E Software
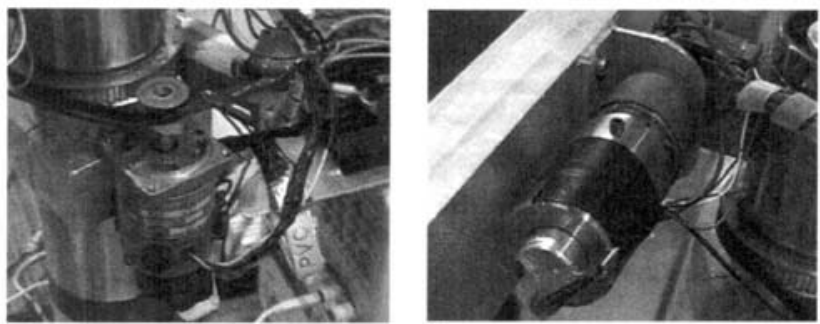

Fig. 4 Photo of assembled DC servomotors: axis 1 (left); and axis 2 (right)

Table 1 Mechanical and electrical specifications of EIHR

\begin{tabular}{|l|c|c|c|c|}
\hline & Gear Ratio & $\begin{array}{c}\text { Encoder } \\
\text { Resolution }\end{array}$ & $\begin{array}{c}\text { Operation } \\
\text { Voltage }\end{array}$ & $\begin{array}{c}\text { Operation } \\
\text { Angle }\end{array}$ \\
\hline Axis 1 & $1: 4.4$ & $512 \mathrm{p} / \mathrm{r}$ & $30 \mathrm{~V}$ & $0-78.9^{\circ}$ \\
\hline Axis 2 & $1: 300$ & $100 \mathrm{p} / \mathrm{r}$ & $12 \mathrm{~V}$ & $0-39.8^{\circ}$ \\
\hline
\end{tabular}

On the other hand, the in-lab designed DSP based EIHR controller is also introduced. The DSP based servo motion controller is designed to drive the DC servomotors with the desired angular velocity. The DSP controller is responsible for the angular position and velocity calculation and PID (proportional-plusintegral-plus-derivative) [10] motion control. Especially, the servo motion controller must be manipulated by the host computer, so that the surgical marker can be tracked in the target window.

The design of image servo tracking control scheme is shown in Fig. 6. The image servo tracking controller is configured in terms of two feedback loops. The inner-control-loop acts as the PID velocity controller, and the velocity feedback is given by

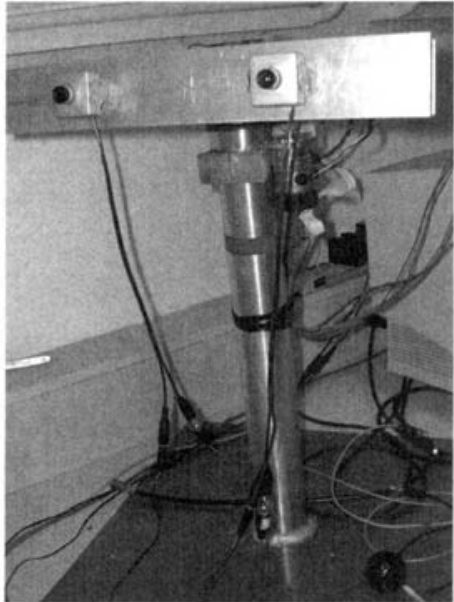

Fig. 5 Photo of assembled EIHR robot with CCD cameras

calculating the motor angular velocity; and the outercontrol-loop acts the image servo tracking controller, and the position feedbacks are given by recognizing the stereoscopic images. Note that the image servo tracking controller will be introduced in the next subsection. The proposed digital PID controller is described as follows:

1 . The angular velocity vector, $\Omega(k)$ is defined as the difference between two sampled angular position vectors, $\theta(k)$ and $\theta(k-l)$, where $T$ is the sampling time. In this work, $T=0.01 \mathrm{sec}$. The $\Omega(k)$ can be expressed as:

$$
\Omega(k)=\theta(k)-\theta(k-1) / T
$$

2. The velocity error vector is defined as the difference between the velocity command vector $r_{v}(k)$ and the actual angular velocity vector $\Omega(k)$, and:

$$
\mathrm{e}_{\mathrm{v}}(k)=\mathrm{r}_{\mathrm{v}}(k)-\Omega(k)
$$

3. By introducing the proportional $\left(\mathrm{K}_{r}\right)$, integral $\left(\mathrm{K}_{e}\right)$, and derivative $\left(\mathrm{K}_{d}\right)$ control gains, the velocity control command vector can be defined as:

$$
\begin{aligned}
u(k)= & u(k-1)+k_{p} e_{v}(k)+ \\
& k_{d}\left[e_{v}(k)-e_{v}(k-1)\right]+k_{t}\left[\sum_{i=1}^{k} e_{v}(i)\right]
\end{aligned}
$$

The test of the proposed PID controller was done for several cases. The DSP based PID motion controller can achieve the desired angular velocity. In this paper, one of them is addressed when $\mathrm{K}_{p}=0.07$, $\mathrm{K}_{d}=0.07$, and $\mathrm{K}_{i}=0.00005$, and the transient response is shown in Fig. 7. 


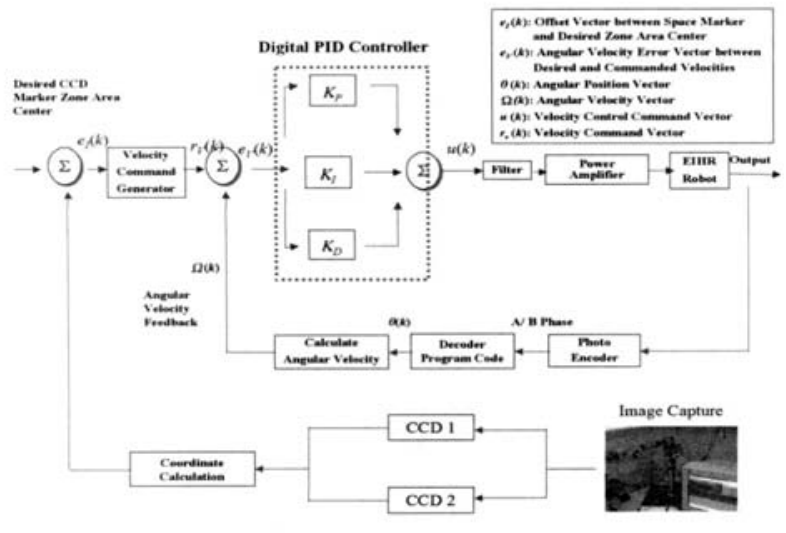

Fig. 6 Image servo tracking control scheme

In addition, the multi-axis control and serial communication interface card was developed using the 89C51 (8051) single processor. This interface card can directly connect to the I/O buses of the DSP controller to achieve the control of multiple axes. Meanwhile, the serial communication interface was also developed to establish the communications between the host computer and the DSP motion controller. The bus layout and control architecture of the proposed multiaxis control and serial communication interface card is shown in Fig. 8. Note that the output bus is a twelve parallel data bit bus, and it can be used to deliver the motion and communication commands; the input bus is an eight parallel data bit bus, and it is used to deliver the host communication message to the DSP controller; the select bus is used to select the communication axis; the DAC (digital-to-analog converter) is used to convert the twelve parallel bits to the analog motor control signal; the MAX 232 is a serial communication interface integrated circuit (IC); and the filter is designed as a low pass filter to avoid high frequency noises.

Finally, the EIHR robot mechanics and control circuit can be combined to promote the image servo tracking based surgical navigation robot, as shown in Fig. 9. In this figure, the analog/digital signal connections and communication interfaces among these devices are addressed. Meanwhile, the photo of in-lab designed hardware components and devices are also shown in this figure. Note that the voltage gain of the linear power amplifier was designed as 10 .

\subsection{Image Recognition of Surgical Marker}

The image recognition is important to the positioning of the surgical marker. In this paper, a three-ball structured surgical marker is designed to simulate the clinical surgical marker. The photos of the

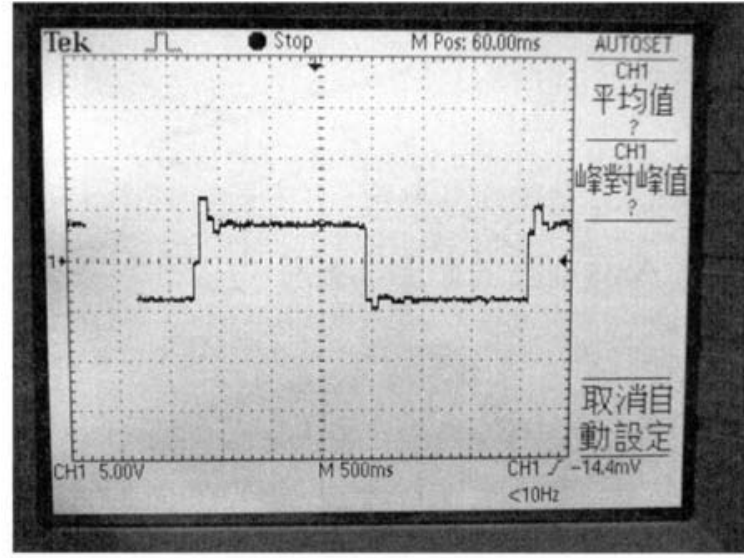

Fig. 7 Transient response of PID controller when $\mathrm{K} \boldsymbol{p}=0.07, \mathrm{Kd}=0.07$, and $\mathrm{K} i=0.00005$

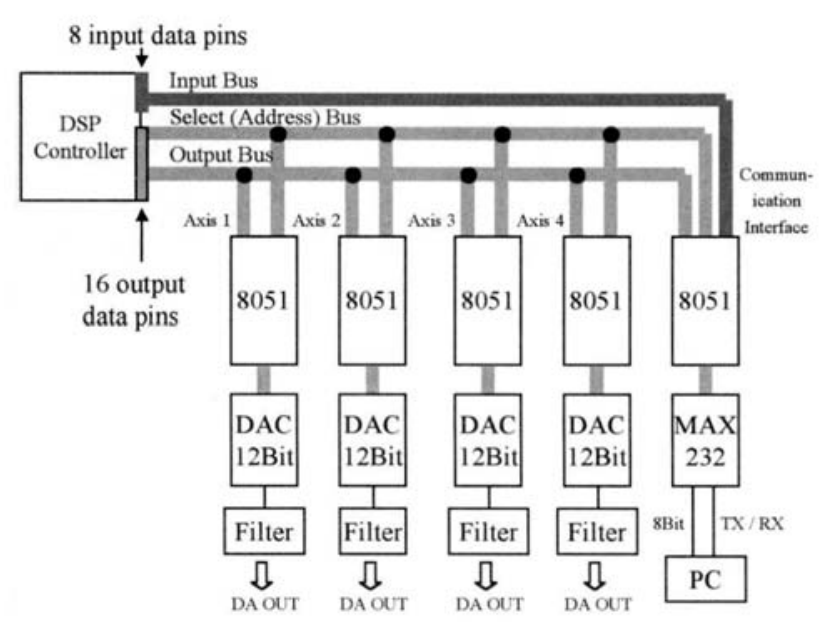

Fig. 8 Bus layout of four-axis control and serial communication interface card

clinical surgical marker [15] and the in-lab designed simulated surgical marker are shown in Fig. 10. The design of three-ball structured surgical marker is based on three space points that are not co-linear forming a unique plane. In this works, three balls forms a triangle structure with non-equal side lengths. The normal vector and the ball centers' plane uniquely determine the space posture of the surgical marker. The surgical marker can be mounted on the hard tissue of the surgical position so that the surgical coordinate can be determined and tracked.

In this work, the stereoscopic images based positioning approach was used. The feature differences between two simultaneously captured stereoscopic images can be used to calculate the space coordinates, and the calculation formulas are introduced in the next subsection. No matter the image capture direction, the 


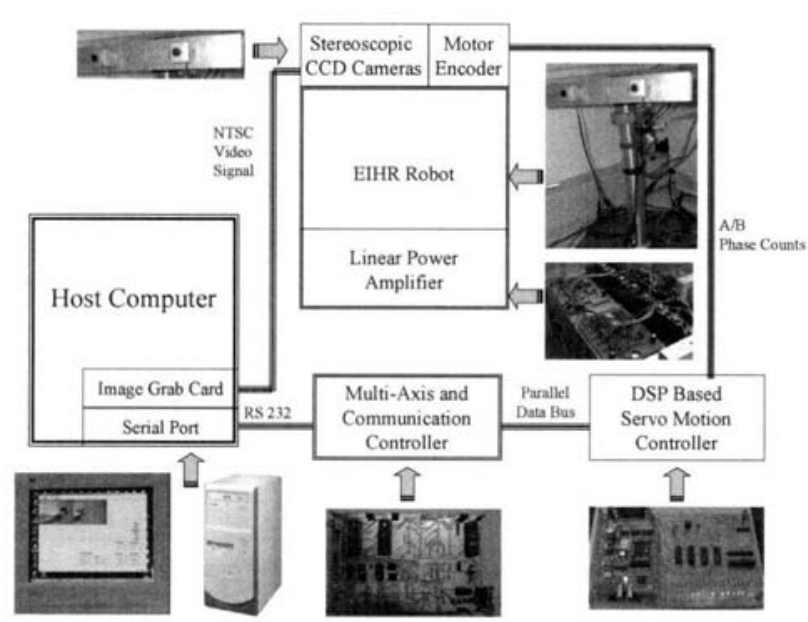

Fig. 9 Signal flow diagram of EIHR navigation robot

ball results in the circle images. Consequently, the recognized ball centers act as the image features of the stereoscopic images, as indicated in Fig. 10 (right figure). Therefore, the image recognition of the surgical marker is to find the two-dimensional image coordinate of the ball centers and radius. Note that the ball's diameter is $38 \mathrm{~mm}$ for the simulated surgical marker.

In most of the clinical application, since the complex image capture environment of the operation room, the fluorescence sensitive or active infrared sensing image capture system is widely used to recognize the surgical marker so that the recognition time and accuracy can be improved. Because this work aims to validate the newly proposed actively tracking based surgical navigation robot, the system prototype does not use the high cost clinical marker. In order to reduce the recognition time and to increase the recognition accuracy, the in-lab designed surgical marker is painted with black color, and the test environment use the white background. Hence, the image capture environment can be similar to the clinical application environment.
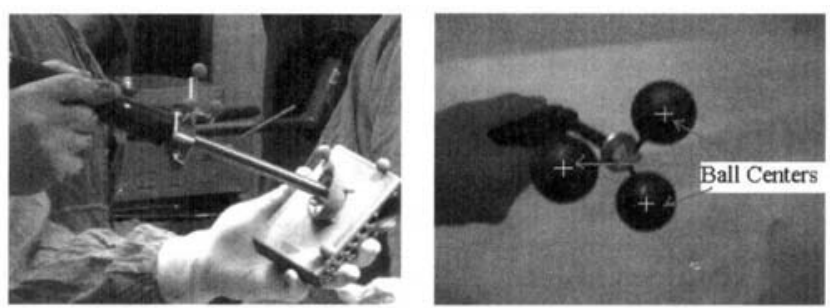

Fig. 10 Photos of clinical surgical marker (left) and in-lab designed simulated surgical marker (right)
Although the applying of black surgical marker and white background reduces the image recognition efforts, the non-uniform lights result in the difficulties in the abstraction of simulated surgical marker. In order to abstract the surgical marker images, the image filtering $[5-6,12]$ is used in the image pre-processing stage, as indicated in Fig. 1. In this work, the Sobel [5] chain code method is used to detect the ball's edge. However, due to the surgical marker is freely moving in space, the image of three balls may be overlapped. This paper discusses four overlap conditions to resolve this problem:

1. Image of three balls are not overlapped with each other, as shown in Fig. 11 (a).

2. Image of two balls are overlapped, and one ball is not overlapped, as shown in Fig. 11 (b).

3. Image of three balls are overlapped, and there are three intersection points, as shown in Fig. 11 (c).

4. Image of three balls are overlapped, and there are four intersection points, as shown in Fig. 11 (d).

Based on the categories indicated in Fig. 11, three balls can be abstracted completely. In addition, due the limited navigation space, the ball radius pixels are limited in a specific range. Such a condition can be used to filter the unreasonable ball recognition, so that the recognition accuracy and reliability can be improved. Consequently, the ball center and radius can be obtained. The ball centers act as the features of the stereoscopic images to calculate the space coordinate of the surgical marker.

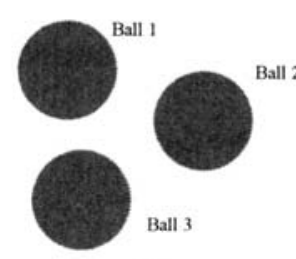

(a)

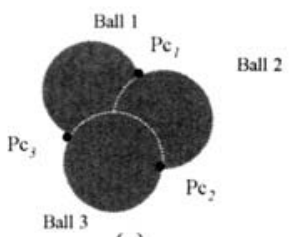

(c)

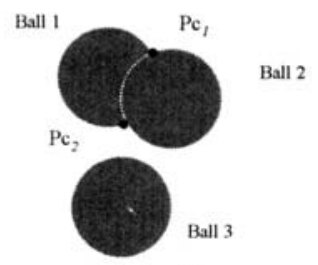

(b)

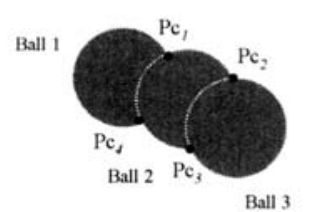

(d)

Fig. 11 Ball image overlap categorization 


\subsection{Fuzzy Logic Based Image Servo Tracking}

The fuzzy system uses the fuzzy set and the membership function to describe the facts with uncertainty [7]. The inference engine is executed according to the input status and knowledgebase to reason the facts and make decision. In this paper, the real-time image servo tracking controller is developed using the fuzzy logic. The fuzzy control model is executed in terms of the fuzzification, rule base categirization, inference engine, and defuzzification, as shown in Fig. 12.

The control goal of the image servo tracking controller is to maintain the simulated surgical marker appeared in the target window of the left CCD camera so that the other CCD camera can also acquire the surgical marker image. Note that if any one CCD camera does not acquire the surgical marker completely, then the space coordinate module cannot work. The target window is defined as a rectangle image sub-area with $120^{*} 160$ image pixels. The target window is located around the right-down corner of the left CCD camera image, as shown in Fig. 13. Therefore, the image servo controller drives the EIHR to track the geometric center of the surgical marker to the target window's center, $(350,240)$, of the left image coordinate.

The image servo tracking controller aims to automatically tack the surgical marker in both of the stereoscopic images. The frequently moving of the EIHR robot affects the quality of acquired images. Therefore, the fuzzy tracking controller works only when the geometric center of the surgical marker exits target window. The proposed fuzzy tracking controller is decoupled into the horizontal and vertical motion models, respectively. Each fuzzy tracking model is defined as two inputs and one outputs fuzzy model. The inputs use the relative distance $\left(\mathrm{L}_{\mathrm{a}}\right.$, in image pixel) and angle ( $\theta_{a}$, in degree) of the surgical marker's

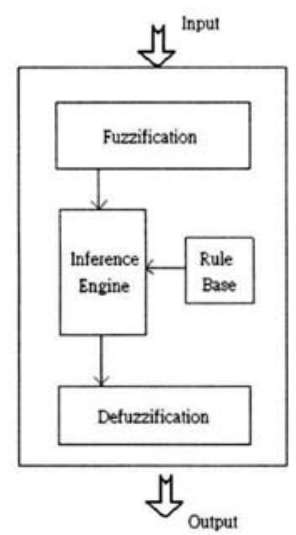

Fig. 12 Fuzzy control system operation diagram

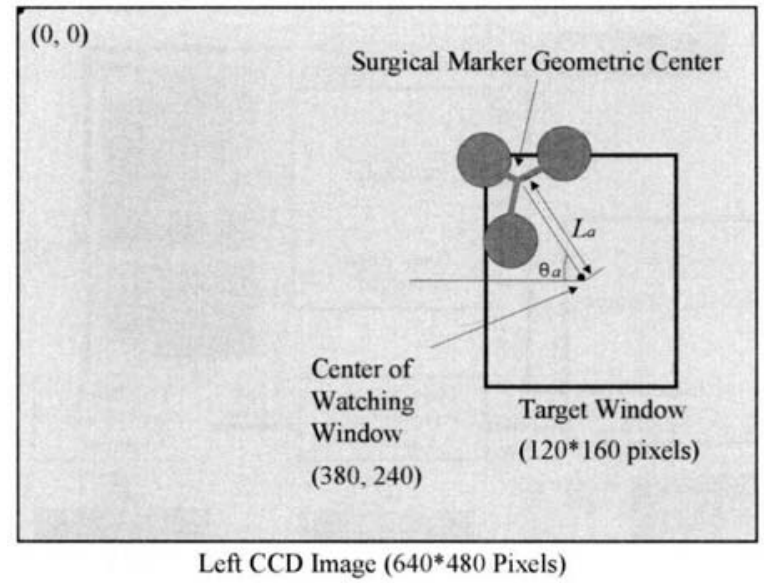

Fig. 13 Target window definition

geometric center to the target window's center, as described in Fig. 13. The output is designed as the motor velocity control command $\left(\mathrm{V}_{\mathrm{a}}\right.$, in pulses/ $0.01 \mathrm{sec})$. The symbols used in the input and output membership functions are listed in Table 2 . The input and output membership functions are shown in Fig. 14.

On the other hand, the rule tables for the horizontal and vertical models can be constructed according to the memberships function and control issues. Table 3 shows the horizontal model rule table. Note that the vertical model [12] can also be constructed in the same manner, and it is not indicated here. Finally, the defuzzification method of center of gravity [7] can be used to determine the actual motor commands.

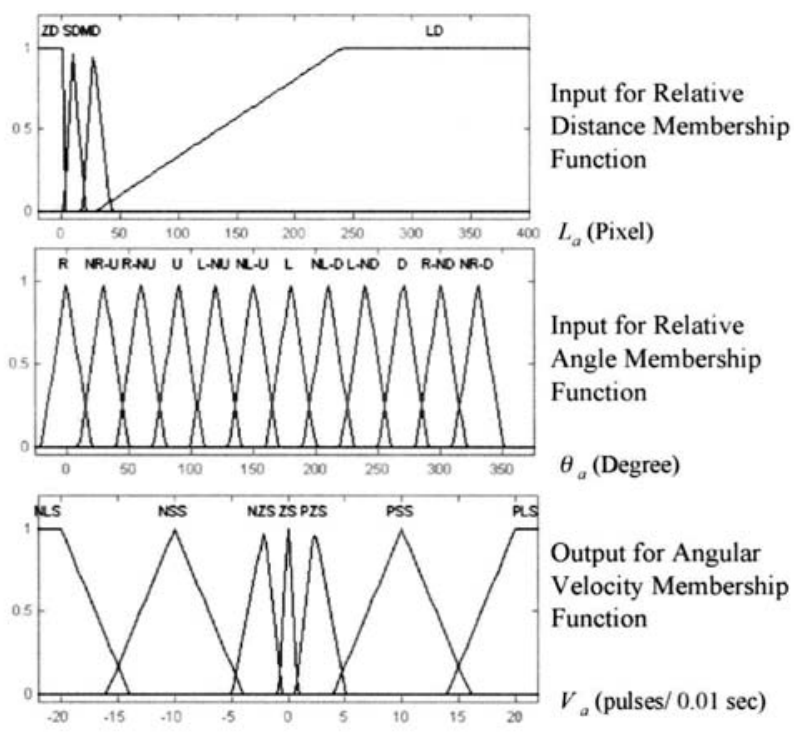

Fig. 14 Input and output membership functions 
Table 2 Symbols used in the input and output membership functions

\begin{tabular}{|c|c|c|c|c|}
\hline & Symbol & Description & Symbol & Description \\
\hline \multirow{2}{*}{$\begin{array}{c}\text { Input } \\
\text { (Distance) }\end{array}$} & LD & Large distance & MD & Medium distance \\
\hline & SD & Smali distance & ZD & Nearly zero distance \\
\hline \multirow{6}{*}{$\begin{array}{l}\text { Input } \\
\text { (Angle) }\end{array}$} & $\mathrm{R}$ & Right direction & NR_U & $\begin{array}{l}\text { Nearly right } \\
\text { direction }\end{array}$ \\
\hline & R_NU & Right and nearly up direction & $\mathrm{U}$ & Up direction \\
\hline & L_NU & Left and Nearly up direction & NL_U & Nearly left and up direction \\
\hline & $\mathrm{L}$ & Left direction & NL_D & $\begin{array}{l}\text { Nearly left and down } \\
\text { direction }\end{array}$ \\
\hline & L_ND & $\begin{array}{l}\text { Left and nearly down } \\
\text { direction }\end{array}$ & $\mathrm{D}$ & \multirow{2}{*}{$\begin{array}{l}\text { Down direction } \\
\text { Nearly right and down } \\
\text { direction }\end{array}$} \\
\hline & R_ND & $\begin{array}{l}\text { Right and nearly down } \\
\text { direction }\end{array}$ & NR_D & \\
\hline & H_NLS & $\begin{array}{l}\text { Horizontal motion with } \\
\text { negative and large speed }\end{array}$ & H_NSS & $\begin{array}{l}\text { Horizontal motion with } \\
\text { negative and small speed }\end{array}$ \\
\hline & H_NZS & \begin{tabular}{|lll}
$\begin{array}{l}\text { Horizontal motion } \\
\text { negative and nearly } \\
\text { speed }\end{array}$ & & zero \\
sped
\end{tabular} & H_ZS & \multirow{2}{*}{\begin{tabular}{|l|}
$\begin{array}{l}\text { Horizontal motion with } \\
\text { zero speed }\end{array}$ \\
$\begin{array}{l}\text { Horizontal motion with } \\
\text { positive and small speed }\end{array}$ \\
\end{tabular}} \\
\hline & H_PZS & \begin{tabular}{|lll}
$\begin{array}{l}\text { Horizontal motion with } \\
\text { positive and nearly } \\
\text { speed }\end{array}$ & \\
\end{tabular} & H_PSS & \\
\hline & H_PLS & $\begin{array}{l}\text { Horizontal motion with } \\
\text { positive and large speed }\end{array}$ & V_NLS & $\begin{array}{l}\text { Vertical motion with } \\
\text { negative and large speed }\end{array}$ \\
\hline & V_NSS & $\begin{array}{l}\text { Vertical motion with } \\
\text { negative and small speed }\end{array}$ & V_NZS & \begin{tabular}{|lll} 
Vertical motion & with \\
negative and nearly & zero \\
speed & &
\end{tabular} \\
\hline & V_ZS & $\begin{array}{l}\text { Vertical motion with zero } \\
\text { speed }\end{array}$ & V_PZS & $\begin{array}{lll}\text { Vertical motion with } \\
\text { positive and nearly } \\
\text { speed }\end{array}$ \\
\hline & V_PSS & $\begin{array}{l}\text { Vertical motion with positive } \\
\text { and small speed }\end{array}$ & V_PLS & $\begin{array}{l}\text { Vertical motion with } \\
\text { positive and large speed }\end{array}$ \\
\hline
\end{tabular}

\subsection{Space Coordinate Calculation}

In this section, the methodology of the space coordinate formula of the surgical marker using the stereoscopic images is introduced. As shown in Fig. 15 , an investigated point such as any of the ball center in space will result in different image coordinates that are measured from two CCD cameras. By comparing the image coordinates of two image frames, the formula for computing the space coordinates can be derived. In Fig. 15, (X1, Y1, Z1) and (X2, Y2, Z2) represents the world coordinate system with respect to CCD 1 and CCD 2 cameras; $\lambda$ is the focus of the CCD camera; B is the optical center distance; $(\mathrm{x} 1, \mathrm{yl})$ indicates the image coordinate of CCD 1; and $(x 2, y 2)$ indicates the image coordinate of $\mathrm{CCD} 2$. Form the geometric relationships,

$$
X_{2}=X_{1}+B
$$


Table 3 Rule table for the horizontal fuzzy tracking model

\begin{tabular}{|c|c|c|c|c|c|}
\hline \multirow{2}{*}{\multicolumn{2}{|c|}{$\begin{array}{c}\text { Speed } \\
\left(V_{a}\right)\end{array}$}} & \multicolumn{4}{|c|}{ Distance $\left(L_{a}\right)$} \\
\hline & & \multirow{2}{*}{$\frac{\mathrm{LD}}{\mathrm{H} \_\mathrm{NLS}}$} & \multirow{2}{*}{$\frac{\mathrm{MD}}{\mathrm{H} \_\mathrm{NSS}}$} & \multirow{2}{*}{$\frac{S D}{H \_N Z S}$} & \multirow{2}{*}{$\frac{\mathrm{ZD}}{\mathrm{H} \_\mathrm{ZS}}$} \\
\hline \multirow{12}{*}{$\begin{array}{l}\text { Angle } \\
\left(\theta_{a}\right)\end{array}$} & $\mathbf{R}$ & & & & \\
\hline & NR_U & H_NSS & H_NSS & H_NZS & H_ZS \\
\hline & R_NU & H_NZS & H_NZS & H_NZS & H_ZS \\
\hline & $\mathrm{U}$ & H_ZS & H_ZS & H_ZS & H_ZS \\
\hline & L_NU & H_PZS & H_PZS & H_PZS & H_ZS \\
\hline & NL_U & H_PSS & H_PSS & H_PZS & H_ZS \\
\hline & $\mathbf{L}$ & H_PLS & H_PSS & H_PZS & H_ZS \\
\hline & NL_D & H_PSS & H_PSS & H_PZS & H_ZS \\
\hline & L_ND & H_PZS & H_PZS & H_PZS & H_ZS \\
\hline & $\mathrm{D}$ & H_ZS & H_ZS & H_ZS & H_ZS \\
\hline & R_ND & H_NZS & H_NZS & H_NZS & H_ZS \\
\hline & NR_D & H_NSS & H_NSS & H_NZS & H_ZS \\
\hline
\end{tabular}

$$
\begin{gathered}
Z_{1}=Z_{2}=Z \\
X_{1}=\frac{x_{1}}{\lambda}\left(\lambda-Z_{1}\right) \\
X_{2}=\frac{x_{2}}{\lambda}\left(\lambda-Z_{2}\right)
\end{gathered}
$$

From combining equations of (4) - (7), the space coordinate relative to CCD camera 1 can be obtained as follows.

$$
\begin{gathered}
X_{1}+B=\frac{x_{2}}{\lambda}(\lambda-Z) \\
Z=\lambda-\frac{\lambda B}{x_{2}-x_{1}}
\end{gathered}
$$

The previous formula can calculate the relative coordinate of the CCD system, however, it is not measured with respect to the EIHR base. The EIHR robot's posture determines the space coordinate of the

$$
\begin{gathered}
Z=(L 2 \sin \alpha+H) \\
X=(L 2 \cos \alpha+L 1) \cos \theta \\
Y=(L 2 \cos \alpha+L 1) \sin \theta
\end{gathered}
$$

surgical marker. The end effector coordinate can be obtained by calculating the forward kinematics [11] of the EIHR. The end effector coordinate relative to the EIHR base, i.e., $(X, Y, Z)$, can be calculated based on the Fig. 16. Note that the arm length and posture angle are indicated in this figure.

Finally, the space coordinate of surgical marker relative to the EIHR base, i.e., $\overrightarrow{S 3}$, can be calculated as in equation (13). Note that $\vec{S} \hat{i}$ and $\overrightarrow{S 2}$ can be obtained from previous equations, as also indicated in Fig. 16.

$$
\overrightarrow{S 3}=\overrightarrow{S I}+\overrightarrow{S 2}
$$




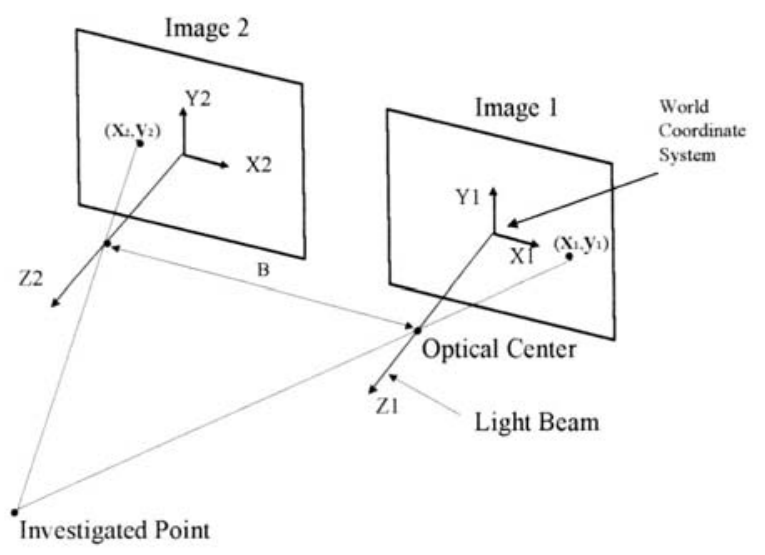

Fig. 15 Space coordinate calculation using stereoscopic images

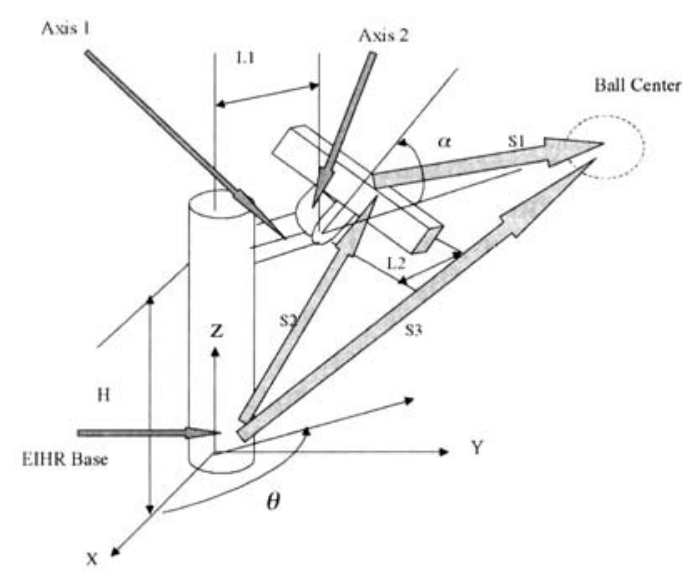

Fig. 16 Kinematics relationship of surgical marker space coordinate vectors

\section{RESULTS}

In this section, the practical results and experimental validation is introduced. The proposed graphical user interface (GUI) is shown in Fig. 17. The proposed GUI provides the stereoscopic images, surgical marker's space coordinate, ElHR robot selftest, ElHR robot control function, 3D digitizer arm space coordinate acquisition, positioning ertor display, calculation time for each cycle, etc.

\subsection{Surgical Marker Recognition and Fuzzy Logic Based Image Servo Tracking}

From previous descriptions, the surgical marker recognition plays an important role to the space coordinate calculation. By using the proposed surgical marker recognition approaches, the ball's centers and

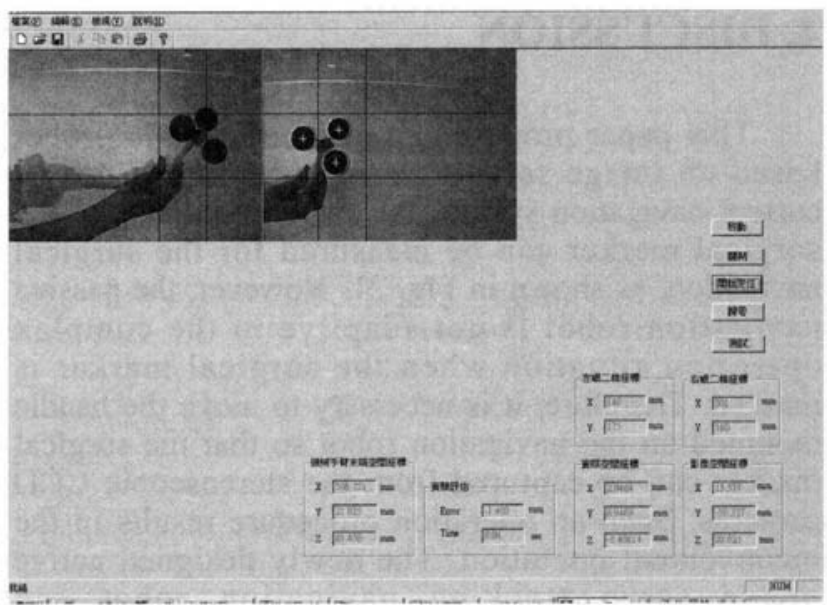

Fig. 17 Graphical user interface of proposed EIHR monitoring program

radiuses can be identified. Fig. 18 shows the circle recognition of three different conditions. The marked white circles are drawn through the obtained center and radius. The overlap of ball images can be abstracted.

In addition, the image servo tracking is also tested in terms of the fuzzy logic control model. In order to test the performance of the fuzzy tracking controller, the geometric centers are simulated at four different image corners, respectively. The results showed that fuzzy tracking controller is possible to tack the geometric centers of surgical marker to the center of the target window, at image pixel position $(380,240)$, as shown in Fig. 19. Fig. 20 shows the photo of image servo tracking experiment.

\subsection{Experimental Evaluation of EIHR Navigation Robot}

Based on the results of surgical marker recognition, the balls' centers can be used calculate the space coordinates. In order to validate the accuracy of the proposed system, a 3D digitizer arm is used to calibrate and validate the proposed system. The 3D digitizer arm is a mechanical space coordinate measurement device, and its accuracy is $0.25 \mathrm{~mm}$. The surgical marker is mounted on the end tip of the 3D digitizer so that the space coordinates of measuring form 3D digitizer and obtaining from the stereoscopic images can be compared simultaneously. The experiment results are shown in Table 4 . The results showed that the average absolute positioning error is about $1.731 \mathrm{~mm}$ within $500 \mathrm{~mm}$ operation range. On the other hand, for all experiments, the image capture and computation time were ranging from 0.03 to 0.05 second for a Pentuim III PC, and the computational efficiency fits the real-time issues. 


\section{DISCUSSION}

This paper proposed an active navigation robot based on image servo tracking techniques. In the current navigation system, the space coordinate of the surgical marker can be measured for the surgical navigation, as shown in Fig. 21. However, the passive navigation robot is not adaptive to the complex operation situation when the surgical marker is masked. Therefore, it is necessary to move the handle mounted on the navigation robot so that the surgical marker can be captured from the stereoscopic CCD cameras. Such an operation procedure results in the inconvenient operation. The newly designed active image servo tracking navigation robot can automatically change its posture to capture the surgical

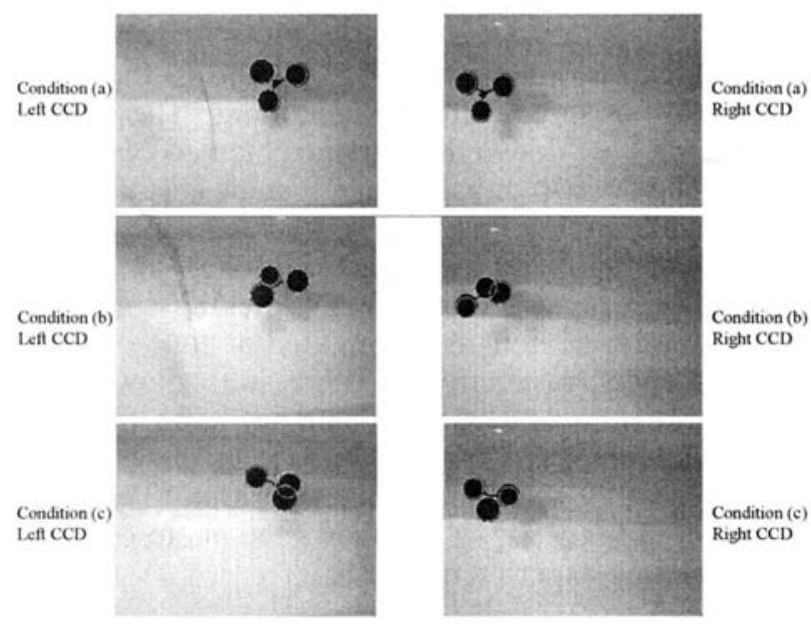

Fig. 18 Surgical marker recognition for three different conditions

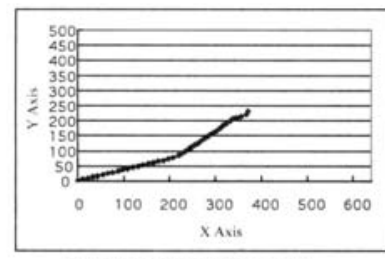

(a) Tracking from left-down concr

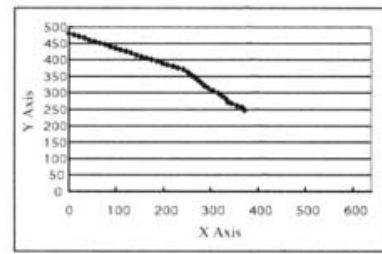

(b) Tracking from lefl-up comer

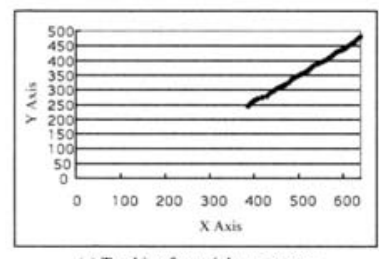

(c) Tracking from right-up comer

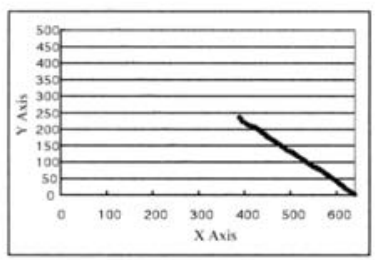

(d) Tracking from right-down comer
Fig. 19 Image servo tracking simulation from four different image corners marker images. It is intelligent and adaptive to the complex operation room environment. Hence, the new design concept is contributed to the CAS systems.

On the other hand, the proposed system is the first generation of this work. From the experiment results of the practical validations of the real-time image servo tracking and the acceptable positioning error of the space coordination, the proposed EIHR navigation robot achieved the desired purposes. In addition, the image capture and computation time were ranging from 0.03 to 0.05 second for all experiments. Such a computational efficiency fits the real-time issues. More specially, the fuzzy logic based intelligent image servo tracking F.IHR robot can automatically adjust its posture to completely capture the images, so that the space coordinate can be calculated accurately.

Currently, the proposed system is developed as a conceptual prototype, and it must be modified according to clinical requirements. The clinical modifications include:

1. Use the clinical marker instead of in-lab designed surgical marker, so that this system can be used in surgery.

2. For the clinical application, the surgical markers are mounting on the patient's hard tissue, fixture too, cutting too, tool guider, etc. Therefore, the image servo tracking algorithm can be modified according to all surgical markers so that all markers can be captured by the stereoscopic CCD cameras. Meanwhile, the image servo tracking controller can be designed to determine the optimal posture to capture high quality mages so that the positioning accuracy can be also improved.

3. The entire navigation system consists of the navigation robot and the navigation software. The navigation software is required to be developed, so that it can couple with the proposed surgical robot to complete the CAS system.

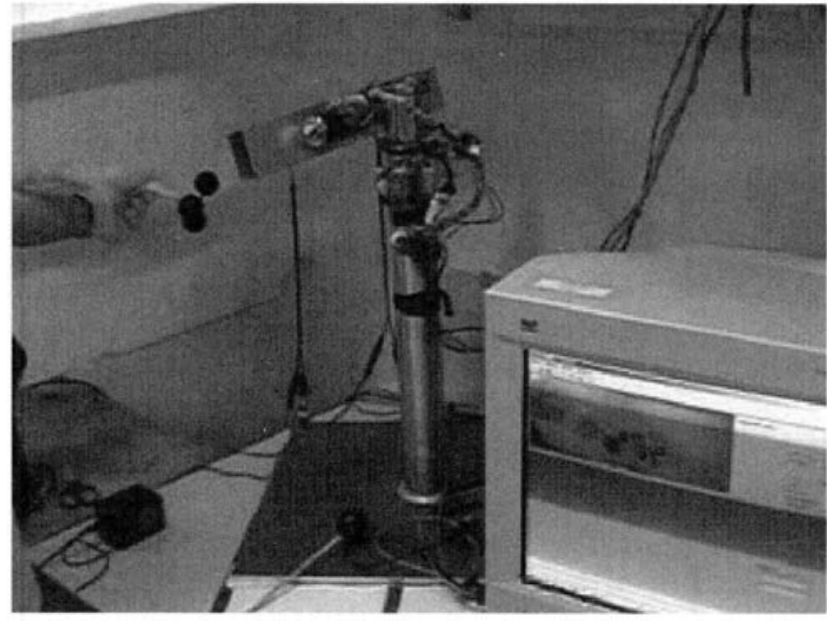

Fig. 20 Photo of image servo tracking experiment 
Table 4 Validation of CCD cameras using 3D digitizer arm

\begin{tabular}{|c|c|c|c|c|c|c|c|c|c|c|}
\hline \multirow{2}{*}{$\begin{array}{l}\text { Experiment } \\
\text { ID }\end{array}$} & \multicolumn{4}{|c|}{ Digitzer Coordinate } & \multicolumn{4}{|c|}{ Image Coordinate } & \multicolumn{2}{|c|}{ Evaluation of Calibration } \\
\hline & $X($ in) & $Y($ in) & $Z(i n)$ & $\begin{array}{c}\text { Distance } \\
(\mathrm{cm})\end{array}$ & $X(\mathrm{~cm})$ & $Y(\mathrm{~cm})$ & $\mathrm{Z}(\mathrm{cm})$ & $\begin{array}{c}\text { Distance } \\
(\mathrm{cm})\end{array}$ & Error $(\mathrm{cm})$ & Error $(\%)$ \\
\hline 1 & 3.519 & 2.914 & -6.918 & 30.02 & 18.518 & -6.975 & 22.957 & 30.31 & -0.29 & -0.95 \\
\hline 2 & 3.493 & 2.931 & -5.959 & 31.66 & 11.624 & 3.565 & 28.831 & 31.29 & 0.37 & 1.18 \\
\hline 3 & 4.056 & 2.463 & -5.788 & 32.25 & 13.15 & -5.224 & 28.928 & 32.20 & 0.05 & 0.15 \\
\hline 4 & 4.157 & 2.778 & -5.154 & 33.87 & 20.004 & -2.268 & 27.311 & 33.93 & -0.06 & -0.17 \\
\hline 5 & 1.16 & 2.735 & -3.341 & 34.04 & 15.577 & -10.327 & 28.623 & 34.18 & -0.15 & -0.44 \\
\hline 6 & 3.949 & 3.385 & -4.374 & 35.69 & 20.415 & -2.807 & 28.937 & 35.52 & 0.16 & 0.46 \\
\hline 7 & 2.847 & 4.425 & -4.112 & 36.11 & 18.889 & -9.048 & 29.636 & 36.29 & -0.18 & -0.50 \\
\hline 8 & 3.746 & 3.894 & -3.734 & 37.22 & 18.211 & -2.047 & 32.654 & 37.44 & -0.23 & -0.61 \\
\hline 9 & 4.713 & 3.251 & -3.382 & 38.43 & 15.735 & 6.093 & 34.361 & 38.28 & 0.15 & 0.39 \\
\hline 10 & 3.392 & 4.534 & -2.913 & 39.14 & 18.368 & -3.037 & 34.407 & 39.12 & 0.02 & 0.06 \\
\hline 11 & 7.69 & 2.154 & -4.262 & 40.50 & 11.046 & -2.852 & 39.132 & 40.76 & -0.26 & -0.64 \\
\hline 12 & 5.907 & 1.226 & -1.208 & 41.64 & 12.655 & -9.744 & 38.175 & 41.38 & 0.25 & 0.61 \\
\hline 13 & 5.907 & 1.226 & -1.208 & 42.73 & 4.724 & -15.407 & 39.628 & 42.78 & -0.05 & -0.11 \\
\hline 14 & 1.786 & -14.732 & -3.705 & 43.19 & 14.014 & -10.127 & 39.803 & 43.40 & -0.21 & -0.48 \\
\hline
\end{tabular}

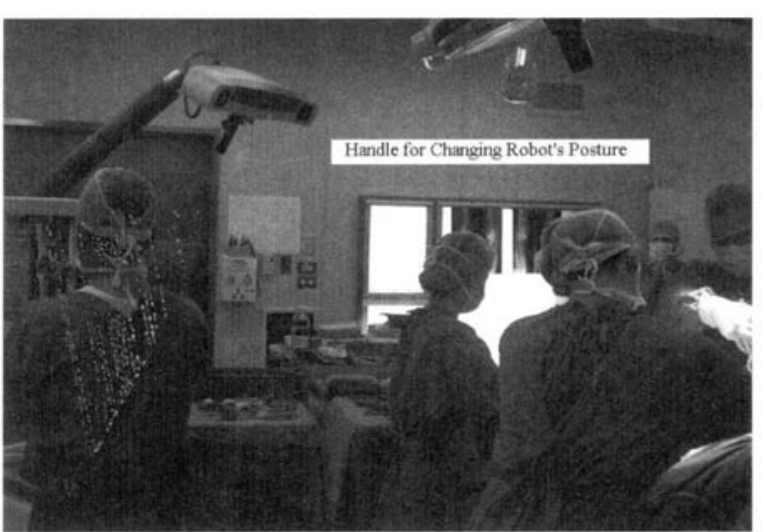

Fig. 21 Navigation robot without image servo tracking function

\section{CONCLUSION}

This paper proposed an active tracking EIHR navigation robot using the stereoscopic image model. The hardware and software components were developed in laboratory using the techniques of mechanical design, DSP based servo motion control, stereoscopic images acquisition, image processing and image recognition, and space coordinate calculation. The practical results include the dual-axis eye-in-hand robot arm, DSP based EIHR motion controller, image capture and recognition module, fuzzy logic based surgical marker tracking module, and surgical marker positioning module. The EIHR robot prototype was validated based on experiment results in terms of the mechanical 3D digitizer. The positioning error is about $1.731 \mathrm{~mm}$ within $500 \mathrm{~mm}$ operation range. The computation time is raging from 0.03 to 0.05 second. In the future, the proposed system will be developed based on the clinical requirements for the real surgical applications.

\section{ACKNOWLEDGEMENT}

This work was partially supported by the National Science Council, Taiwan, R.O.C., under Grants NSC 91-2212-E-182-001

\section{REFERENCES}

1. W. Birkfellner, K. Huber, A. Larson, D. Hanson, M. Diemling, P. Homolka, and H. Bergmann, "A Modular Software System for Computer-Aided Surgery and Its First Application in Oral Implantology," IEEE Transaction on Medical Imaging, Vol. 19, No. 6, 2000.

2. R. Chassaing, Digital Signal Processing with $C$ and the TMS320C30, Wiley-Interscience, 1992.

3. T. Dohi, Y. Ohta, M. Suzuki, K. Chinzei, T. Horiuchi, D. Hashimoto, M. Tsuzuki, "Computer Aided Surgery System (CAS): Development of Surgical Simulation and Planning System with Three Dimensional Graphic Reconstruction," Proceedings of the First Conference on Visualization 
in Biomedical Computing, pp. 458-462, 1990.

4. P.J. Edwards, A.P. King, C.R. Maurer, D.A. de Cunha, D.J. Hawkes, D.L.G. Hill, R.P. Gaston, M.R. Fenlon, A. Jusczyzck, A.J. Strong, C.L. Chandler, and M.J. Gleeson, "Design and Evaluation of a System for Microscope-Assisted Guided Interventions (MAGI)," IEEE Transaction on Medical Imaging, Vol. 19, No. 11, 2000.

5. R.C. Gonzalez and R.E. Woods, Digital Image Processing, 2nd Edition, Prentice-Hall, 2002.

6. Ramesh Jain, Rangachar Kasturi, Brain G. Schunck, Machine Vision, McGRAW-HILL International Edition, 1995.

7. J.S.R. Jang, C.T. Sun, and E. Mizutani, Neuro-Fuzzy and Soft Computing- A Computational Approach to Learning and Machine Intelligence, Prentice Hall, 1997.

8. D.J. Kruglinski, Inside Visual C++ - The Standard Reference for Programming with Microsoft Visual C++ Version 4, Washington:Microsoft, 1996.

9. C.H. Kuo, M.Y. Lee, C.C. Huang, K.F. Hung and Y.S. Chiu, "Development of 3D Navigation System for Retained Auricular Prosthesis Application," Journal of Medical and Biological Engineering, Vol. 23(3), pp. 149-458, 2003.
10. C.L. Phillips and R.D. Harbor, Feedback Control Systems, Prentice-Hall, 2000.

11.M.W. Spcon and M. Vidyasagar, Robot Dynamics and Control, John Wiley \& Song, 1989.

12.Y.L. Tsai, Development of 3D Localization Using Stereoscopic Image Processing, Master Thesis, Chang Gung University, 2003.

13.C3x Super DSK User's Guide, Texas Instrument Incorporated, 1999.

14.TMS320C3x DSP Starter Kit User's Guide, Texas Instrument Incorporated, 1996.

15.VectorVision - Hip Navigation, BrainLab AG Germany, 2002

16. www.ni.com 